\title{
antibody repertoire
}

5 Tianling $\mathrm{Ou}^{1 *}$, Wenhui $\mathrm{He}^{1 *}$, Brian D. Quinlan ${ }^{1}$, Yan $\mathrm{Guo}^{1}$, Pabalu Karunadharma ${ }^{2}$, Hajeung

6 Park ${ }^{3}$, Meredith E. Davis-Gardner ${ }^{4}$, Mai H. Tran ${ }^{1}$, Yiming Yin ${ }^{1}$, Xia Zhang ${ }^{1}$, Haimin Wang ${ }^{5}$, Guocai Zhong ${ }^{5,6}$, Michael Farzan ${ }^{1}$

$10{ }^{1}$ Department of Microbiology and Immunology, The Scripps Research Institute, Jupiter, FL

1133458 USA

$12{ }^{2}$ Genomics Core, The Scripps Research Institute, Jupiter, FL 33458, USA

$13{ }^{3}$ X-ray Crystallography Core, The Scripps Research Institute, Jupiter, FL 33458 USA

$14{ }^{4}$ The Yerkes National Primate Research Center, Emory University, Atlanta, GA 30329 USA

$15{ }^{5}$ Institute of Chemical Biology, Shenzhen Bay Laboratory, Shenzhen, China

$16{ }^{6}$ School of Biology and Chemical Biology and Biotechnology, Peking University Shenzhen

17 Graduate School, Shenzhen, China

18 *These authors contributed equally

19 Correspondence: mfarzan@scripps.edu

20

21 Keywords: Cas12a, B-cell receptor, HCDR3, HIV-1 V2-glycan antibody, Mb2Cas12a, PG9, 22 CH01, SOSIP 


\section{Abstract}

B cells have been engineered $e x$ vivo to express an HIV-1 broadly neutralizing antibody

(bNAb). B-cell reprograming may be scientifically and therapeutically useful, but current approaches limit B-cell repertoire diversity and disrupt the organization of the heavy-chain locus. A more diverse and physiologic B-cell repertoire targeting a key HIV-1 epitope could facilitate evaluation of vaccines designed to elicit bNAbs, help identity more potent and bioavailable bNAb variants, or directly enhance viral control in vivo. Here we address the challenges of generating such a repertoire by replacing the heavy-chain CDR3 (HCDR3) regions of primary human $B$ cells. To do so, we identified and utilized an uncharacterized Cas12a ortholog that recognizes PAM motifs present in human JH genes. We also optimized the design of 200 nucleotide homology-directed repair templates (HDRT) by minimizing the required 3'-5' deletion of the HDRT-complementary strand. Using these techniques, we edited primary human B cells to express a hemagglutinin epitope tag and the HCDR3 regions of the bNAbs PG9 and CH01. Those edited with bNAb HCDR3 efficiently bound trimeric HIV-1 antigens, implying they could affinity mature in vivo in response to the same antigens. This approach generates diverse B-cell repertoires recognizing a key HIV-1 neutralizing epitope. 


\section{INTRODUCTION}

42 Traditional vaccination approaches do not elicit broadly neutralizing antibodies (bNAbs) that

43 target conserved epitopes of HIV-1 envelope glycoprotein trimer (Env). ${ }^{1-4}$ Human precursor B-

44 cell receptors (BCRs) that can develop into bNAbs are rare, ${ }^{5,6}$ and mature bNAbs have

45 properties that are difficult to access through antibody maturation. ${ }^{1,7,8}$ A number of groups have

46 begun to explore an alternative to conventional vaccines in which B cells themselves are

47 reprogrammed. ${ }^{9-13}$ This approach employs CRISPR-mediated editing of the BCR loci so that the

48 edited B-cell expresses a mature HIV-1 bNAb. In addition to its long-term potential for

49 reprograming human immune responses, BCR editing can be applied more immediately to

50 generate animal models useful for assessing vaccination strategies, and for developing more

51 potent and bioavailable bNAb variants.

52 The BCR includes a membrane-bound heavy chain $(\mathrm{H})$ covalently associated with a light chain

53 (L). Both chains are composed of a variable and a constant region. The heavy-chain variable

54 domain is formed by a process of VDJ recombination of the immunoglobulin heavy chain $(\operatorname{IgH})$

55 gene. In humans, one of the 38-46 functional variable (VH) genes recombines with one of 23

56 diversity $(\mathrm{DH})$ and one of 6 joining $(\mathrm{JH})$ genes. ${ }^{14}$ The recombination process also introduces

57 diversity at the junctions of $\mathrm{VH}, \mathrm{DH}$, and $\mathrm{JH}$ genes through removal and addition of nucleotides.

58 The light-chain variable domain is formed similarly by VJ recombination of the IgL gene. The

59 naïve B-cell repertoire thus reflects extensive combinatorial diversity. ${ }^{15-18}$ This diversity is

60 further amplified after antigen exposure. B cells undergo somatic hypermutation (SHM) as they

61 compete for access to antigen in the lymph-node germinal centers, a process resulting in affinity

62 maturation of the BCR. ${ }^{19}$ 
63 The combinatorial diversity of the B-cell repertoire complicates efforts to reprogram BCR. To

64 date, investigators have bypassed this challenge by targeting an unvarying intron between the recombined variable region and the $\operatorname{IgM}$ constant region $(\mathrm{C} \mu) .{ }^{9}, 11-13$ This strategy introduces a

66 single cassette encoding an exogenous promoter and bNAb heavy- and light-chain sequences

67 into this heavy-chain intron. By design, these constructs halt expression of the native variable

68 heavy chain. Expression of the native B-cell light chain is usually also prevented through various mechanisms. While powerful and convenient, this approach eliminates combinatorial diversity and relies solely on SHM to broaden the HIV-1 neutralizing response. In addition, it introduces several less physiologic elements including novel locations for both variable genes, use of exogenous promoter, and some architectural differences between the expressed bNAb-like

73 construct and native antibodies. These limitations may be especially important if edited B cells

74 need to adapt efficiently to a diverse HIV-1 reservoir, ${ }^{20,21}$ or when the edited repertoire is used to 75 study B cell biology. ${ }^{22}$

76 Here we develop a complementary approach in which sequence encoding a bNAb HCDR3 is

77 introduced into a diverse BCR repertoire at its native location. This approach is useful with

78 antibodies that are highly dependent on the HCDR3 to bind antigen, including members of an

79 exceptionally potent class of bNAbs that recognize the Env apex/V2-glycan epitope. ${ }^{23,24}$

80 However, retaining combinatorial diversity in a natural B-cell setting poses several challenges.

81 First, long homology arms of a homology-dependent repair (HDR) template (HDRT) can

82 overwrite the V-encoded region of a heavy chain. Second, the region 5' of the HCDR3-encoding

83 region is necessarily diverse, and thus editing can be variably efficient due to mismatch of a

84 homology arm with its chromosomal complement. Third, introducing exogenous HCDR3

85 requires deletion of chromosomal material of unknown length and content, rather than simply 
insertion of sequence, or direct replacement of a known sequence. To address these challenges, we identified a previously uncharacterized Cas 12 a variant ${ }^{25}$ that efficiently recognizes a specific four-nucleotide protospacer adjacent motif (PAM) present in the 3' region of the most commonly used JH genes in humans. We also optimized the use of 200-nucleotide (nt) single-stranded HDRT with short (50 nt) homology arms, demonstrating in the process that editing efficiency is primarily determined by the length of a 3 ' mismatch tail rather than the relationship of the HDRT to transcription direction (sense or antisense) or to the target strand of the CRISPR guide RNA (gRNA). With these procedures, we altered the specificity of primary human B cells by editing their HCDR3 regions to bind HIV-1 Env, while retaining the original diversity of the VH repertoire. These studies demonstrate the feasibility of an alternative approach to human B-cell reprogramming.

97

\section{RESULTS}

Targeting a conserved region of the immunoglobulin heavy chain locus with a Cas12a

\section{ortholog}

A major challenge of precisely replacing the HCDR3-encoding region of a diverse primary B cell population is the variability of the mature $\mathrm{IgH}$ locus. This variability arises from the random combinations of $\mathrm{V}, \mathrm{D}$, and $\mathrm{J}$ segments which are joined imprecisely and unpredictably. ${ }^{16}$ It complicates two processes necessary for CRISPR-mediated editing of the B-cell locus, namely the selection of a guide RNA (gRNA) that must complement a 20-24 nucleotide genome

106 sequence, and the design of HDRT whose 5' and 3' homology arms must complement even

107 longer genomic regions. ${ }^{26-28}$ 
We began by designing a gRNA that recognizes a large proportion of $\mathrm{BCR}$ and targets genome cleavage to site of insertion, where it is most efficient. The HCDR3 is encoded by of the 3' end of a VH gene, a DH gene, and the 5' end of a JH gene (Figure 1A). There are six human JH segments, and a JH4 alone participates in $50 \%$ of productive human VDJ-recombination events. ${ }^{29,}{ }^{30}$ Due to junctional diversity, the $3^{\prime}$ 'JH region is conserved, but the 5' is less predictable. However, the 3' of JH4 did not contain any canonical Cas 12a PAM $^{31}$ sequences (TTTV), and the available Cas9 PAM (NGG) ${ }^{32}$ mediates cleavage too distal from the site of insertion. Instead, two potential non-canonical Cas12a PAM sites, GTTC and TTCC, ${ }^{33}$ were well positioned to facilitate gRNA recognition of conserved $\mathrm{JH}$ regions while cleaving where a new HCDR3 would be inserted. We therefore characterized a number of Cas12a orthologs for their ability to recognize these divergent PAM sites. To do so, we tested several uncharacterized Cas12a orthologs 25 in the human B-cell line Jeko-1. Jeko-1 cells were transfected with plasmids encoding BsCas12a, TsCas12a, Mb2Cas12a, or Mb3Cas12a along with plasmids encoding gRNAs adjacent to the GTTC and TTCC PAM regions. DNA cleavage within the HCDR3 frequently results in error-prone nonhomologous end joining (NHEJ) that eliminates expression of the Jeko-1 BCR. ${ }^{34}$ Thus loss of IgM expression indicates a successful double-strand break. Among the Cas12a orthologs tested, Mb2Cas12a most efficiently cleaved the Jeko-1 JH4 region initiated with GTTC and TTCC, with the highest efficiency (18.9\%) observed when the GTTC PAM was targeted (Figure 1B).

Ribonucleoprotein (RNP) forms of CRISPR effector proteins, electroporated into cells, are typically more efficient than plasmids expressing the same protein. ${ }^{28,35} \mathrm{We}$ accordingly produced Mb2Cas12a RNP and compared its editing efficiency with a commercial AsCas12a RNP in Jeko-1 cells, again as determined through loss of IgM expression. These RNP cleaved a 
canonical Cas12a TTTG with comparable efficiency but Mb2Cas12a cleaved three noncanonical PAM regions more efficiently (Figures $1 \mathrm{C}$ and 1D), demonstrating that Mb2Cas12a has a broad PAM specificity and efficiently edits the HCDR3 region of Jeko-1 cells. Notably, Mb2Cas12a RNP efficiently cleaved JH4 when initiated with a GTTC PAM, and this PAM region is conserved in the JH-genes of both humans and rodents.

\section{Optimization of gene editing using single-stranded HDR templates}

We also optimized the design of HDRT used to replace a native HCDR3 region. Again, the underlying diversity of the recombined heavy-chain limited our options. Most importantly, the HDRT homology arms needed to remain short to maximize complementarity to the $3^{\prime} \mathrm{VH}$ region. We therefore optimized a strategy based on short single-stranded DNA (ssDNA) HDRT with 50-nucleotide homology arms by monitoring the efficiency with which a hemagglutinin (HA) tag could replace the Jeko-1 HCDR3 region. Specifically, we compared sense and antisense forms of two distinct HDRT, each with different length linkers bounding the HA tag, and cleavage at four distinct Mb2Cas12a sites and four proximal SpCas9 sites (Figure 2A and Figure S1). Note that some of these sites are unique to the Jeko-1 HCDR3 region, and are therefore not generalizable to primary B cells. Knock-in efficiency was determined by flow cytometry with fluorescently labelled anti-HA antibodies (Figure 2B). We observed that on average, with four different cut sites and four distinct HDRT, Mb2Cas12a and SpCas9 edited with comparable efficiencies (Figure 2C). We analyzed these same data by comparing a number of parameters proposed to impact editing efficiencies in other systems. ${ }^{27,28,36,37}$ However, no significant differences were observed when sense or anti-sense strand HDRT were used (Figure 2D), and only a modest difference for Mb2Cas2, but not for SpCas9, was observed when target (complementary to gRNA) or non-target strand HDRT was used (Figure 2E). These data suggest 
that our system is distinct from those of previous studies, perhaps because both DNA deletion and insertion are required to replace an HCDR3.

Figure $3 \mathbf{A}$ presents a model of a system in which deletion of chromosomal DNA and insertion of novel sequence are both necessary for successful editing. After CRISPR-mediated formation of a double-strand break, HDR is initiated by 5' to 3' resection of DNA, exposing two 3' ends, one of which can anneal to a single-stranded HDRT homology arm. However, at least one 3' end necessarily includes sequence that must be deleted 3' to 5', either on the HDRT-complementary strand, creating a 3' mismatch tail, on the opposing strand, or on both. As shown in Figures 3B and $3 \mathrm{C}$, the length of this 3 'mismatch tail strongly predicts editing efficiency in this system regardless of whether the double-stranded break was mediated by Mb2Cas12a or SpCas9.

164 Specifically, editing is significantly more efficient when mismatch tails are shorter than 10 nucleotides, presumably because longer tails prevent polymerase priming and templated extension of the 3' arm. We presume that this principle can be extended to other systems in which a templated sequence must replace a chromosomal region by HDR.

\section{Reprogramming B cell specificity towards HIV through HCDR3 replacement}

Using the strategies described above, we then designed HDRT that could replace the Jeko-1

HCDR3 with those of PG9 or PG16 (Figure 4A), two potent HIV bNAbs directed against a V2

171 apex of the HIV-1 Env trimer. ${ }^{38,39}$ To monitor the successful introduction of these HCDR3 in

172 contexts where the resulting BCR does not bind soluble native-like HIV-1 Env trimer (SOSIP) ${ }^{40}$,

$173{ }^{41}$, we employed an antibody, PSG2, ${ }^{42}$ that recognizes sulfated tyrosines present at the tips of the

174 PG9 and PG16 HCDR3. In addition, we monitored HIV-1 Env binding with two reagents, a

175 SOSIP protein derived from the HIV-1 isolate BG505, and the multivalent nanoparticle (E2p) ${ }^{43}$

176 based on the same BG505 HIV-1 isolate. Jeko-1 cells were edited with HDRT-PG9-CVR and 
177 HDRT-PG9-CAR to express two forms of the PG9 HCDR3, distinguished by an alanine or 178 valine immediately adjacent to the HCDR3-initiating cysteine. Cells edited with either HDRT could be recognized by all three binding reagents, indicating that the resulting BCR could bind the BG505 Env (Figures 4B and C). In contrast, Jeko-1 cells edited to express the PG16 HCDR3 were recognized only by PSG2, indicating that editing was efficient, but that the resulting BCR did not bind HIV-1 Env. As expected, none of these three antigens bound Jeko-1 cells in which a control HDRT, introducing an HA-tag into the HCDR3, was employed.

We further characterized Jeko-1 cells edited with HDRT-PG9-CAR by enriching edited cells by

FACS with the PSG2 antibody (Figure 4D). Sorted cells were then analyzed by flow cytometry

Figure 4 indicate that the HCDR3 of Jeko-1 cells can be replaced by that of PG9 to generate a 
The preceding studies showed that Mb2Cas12a could cleave a conserved region of the JH4 gene useful for introducing an exogenous HCDR3 sequence, that editing with sense-strand HDRT in this setting is optimal because it minimizes the length the 3' mismatch tail, and that the PG9 HCDR3 could function with the divergent Jeko-1 heavy- and light-chain to bind multiple HIV-1 Env trimers. However, primary human B-cells pose an additional challenge: in contrast to Jeko-1 cells, the VH-gene sequences of primary cells are variable and unpredictable. This difficulty complicates the design of the 5' homology arm, which must complement the 3 ' region of the $\mathrm{VH}$ gene. Alignment of the 3' regions of the most commonly used VH gene families, namely VH1, VH3, and VH4 (Figure S2) revealed that a good deal of interfamily diversity, but showed that intrafamily diversity was limited among the 3' nucleotides. We accordingly evaluated HDRT with $5^{\prime}$ homology arms based on consensus sequences for each of these VH families in primary human B cells. These cells were isolated from peripheral blood and activated by an anti-CD180 antibody $^{11}$ for 48 hours before electroporation with Mb2Cas12a RNP along with different HDRTs. Editing efficiency was measured 48 hours post electroporation by flow cytometry using the anti-sulfotyrosine antibody PSG2 (Figure 5A). Two different fluorophores were used to label PSG2 to eliminate non-specific binding from either fluorophore. As negative controls, primary human primary B cells were activated in the same way, but they were not electroporated (null), or electroporated with Mb2Cas12a RNP and an HDRT that was not homologous to any sequence in the human genome. The HDRT homologous to VH1 had relatively lower efficiency than to the other two families, largely due to the lower VH1 usage frequency in mature human B cells (Figure 5B). A equal mixture of three HDRTs (those of VH1, VH3, and VH4) edited more cells than any individual HDRT, suggesting a diverse pool of B cells could be targeted simultaneously. NGS performed on two sets of B cells from different donors edited with the 
222 mixed HDRT, and the frequency of successful in-frame editing reflected the efficiencies of the individual HDRT (Figure 5C). These data show that, using consensus HDRT homology arms, approximately $1 \%$ of primary human B cells can be edited to express the PG9 HCDR3.

\section{The diversity and specificity of HCDR3-edited primary human B-cell repertoires}

To determine if HCDR3-edited BCR acquired their reprogrammed specificity and retained their

VH diversity, edited B cells were expanded for a week and then sorted with an appropriate

RNP were used to cleave the JH4 region, and mixtures of three HDRT, recognizing consensus 3'

sorted with an anti-HA antibody (Figure 6A) or the CRF250 SOSIP trimer derived from the 
245 6F). Notably, antibodies generated from the VH3-30 gene bound SOSIP trimers and neutralized

246 HIV-1 more efficiently than those based on VH3-33, indicating that VH3-30 and perhaps several

247 other VH genes could serve as alternative starting points for PG9-like antibodies. Collectively,

248 Fig. 6 shows that diverse human B cells can be edited to express the PG9 HCDR3, conferring on 249 many of these cells the ability to bind a SOSIP trimer and express antibody primed to neutralize 250 multiple HIV-1 isolates.

\section{DISCUSSION}

253 Transgenic mice engineered to expressed human variable-chain sequences of bNAbs and

254 inferred germline forms of these bNAbs have been used extensively to study bNAb maturation in

255 response to HIV-1 antigens. ${ }^{44-46}$ These mice were developed primarily to study vaccination

256 strategies, but they could potentially be used to improve the breadth and potency of bNAbs as

257 well. The advent of CRISPR technologies enables ex vivo editing of mature B cells, and adoptive

258 transfer edited B cells into a new murine host. ${ }^{9,11-13}$ CRISPR-mediated editing of B cells is more

259 rapid and versatile than developing a transgenic mice, but a more limited subset of B cells

260 express the bNAb of interest. These cells can nonetheless be amplified through vaccination, and

261 they undergo class switching and SHM. As this technology advances, reprogramming of mature

262 naïve B cells could replace transgenic mice as a means of evaluating and optimizing vaccine

263 protocols. This approach could also be developed as an in vivo alternative to phage- and yeast-

264 display technologies to improve the breadth, potency, or bioavailability of existing antibody or

265 even another biologic. Finally, this technology may form the basis of future vaccines and cell

266 therapies, following a path established by chimeric antigen-receptor (CAR) T cells. 
267 Nearly every reported effort to date to reprogram primary B cells utilizes a conserved intron downstream of the VDJ-recombined variable region. ${ }^{9,11-13}$ A typical insertion cassette initiates with a poly(A) tail to terminate transcription of the native variable region, followed by an exogenous promoter, a human bNAb light-chain variable-region sequence, a P2A peptide or

271 linker, and a heavy-chain variable-region sequence with a splice donor that promotes splicing to

272 native constant genes. This approach is efficient because every mature B cell could theoretically

273 be modified, because HDRT with long homology arms can be used, and because a single editing

274 event introduces both variable chains at once. This efficiency makes this approach attractive to

275 most investigators, but other approaches that preserve the organization of the heavy-chain locus

276 have been attempted. For example, Voss et al. ${ }^{10}$ have explored an alternative in which the entire

277 native variable-region was replaced by the heavy-chain variable of the bNAb PG9, before

278 reverting to the more common intron-targeting approach.

279 A common property of all previous investigations is that an entire heavy chain or heavy-

280 chain/light-chain pair are introduced. Thus initially B cells express a monoclonal antibody which

281 then can diversify through SHM. As a consequence, one major contributor to antibody diversity,

282 namely combinatorial diversity, is bypassed. Such an approach is necessary for many HIV-1

283 bNAbs because antigen recognition is distributed across multiple CDR loops. However most

284 known V2-glycan/apex bNAbs have long, acidic HCDR3 regions that make an unusually large

285 contribution to Env recognition. Moreover, neutralization with these antibodies is especially

286 potent, typically ten-fold higher than with other classes of bNAbs. Finally, antibodies of this

287 class, uniquely recognizing a quaternary epitope, are especially sensitive to the quality of HIV-1

288 antigens. We therefore undertook to reprograming human B-cells to express the HCDR3 of a 289 potentV2-glycan/apex antibody while largely preserving the combinatorial diversity of the 
290

291

292

293

294

295

296

297

298

299

300

301

302

303

304

305

306

307

308

309

310

311

312

reservoir. Ultimately, we anticipate this diversity will enhance and personalize the adaptive humoral response to the diversity of HIV-1 isolates in reservoirs of infected humans.

However, before this concept can be tested, we had to address several challenges unique to introducing an HCDR3 into primary human B cells. These challenges arose from two sources.

First, for optimal editing, a double-strand break should be introduced near the insert region, in this case at the 5' of a commonly used J gene such as JH4. However, due to junctional diversity, this region is highly variable in a diverse repertoire. Second, HDRT with long homology arms are typically more efficient, but long arms would complement only a narrow BCR subsets or overwrite the native $\mathrm{VH}$ gene.

To address the first challenge, we initiated studies with the CRISPR effector protein Cas 12a. We began with Cas12a because, unlike the more commonly employed Cas9, this CRISPR effector protein cleaves distally from its PAM and seed regions. Thus a more variable region can be cleaved from a more predictable gRNA target sequence. However, most commonly studied Cas12a orthologs, including LbCas12a and AsCas12a, use restrictive PAM recognition sequences $^{31}$ absent in human JH4 sequences. We therefore characterized a number of less studied Cas12a variants, and identified one, MbCas12a, that efficiently recognized a GTTC PAM present at an optimal location in both human and rodent JH4 genes. Thus electroporated Mb2Cas12a RNP efficiently introduced double-strand breaks near the 5' of the JH4-encoded region in Jeko-1 cells and in primary human B cells.

Our second challenge arose from the unpredictability of the $\mathrm{VH}$-encoded region in the diverse repertoire of primary B cells, precluding the use of long HDRT. We accordingly designed HDRT that recognized short consensus sequences at the 3' of three VH-gene families. We also optimized the efficiency of editing using these shorter HDRT. To do so, we first evaluated the 
313 impact of two parameters that have been proposed to alter editing efficiency. First, we asked

314 whether the HDRT should complement the coding or non-coding sequence, and we also

315 investigated whether it should complement the gRNA target strand or its opposite. Both

316 parameters have be reported to contribute to editing efficiencies of other systems, but neither of

317 these variables had a dramatic impact on editing efficiency in our study. Further analysis

318 identified a distinct, decisive factor in editing efficiency, namely the length the 3' mismatch tail.

319 While 5'-3' resection is among the first events in HDR, removal of 3' end is rate limiting. Our

320 data suggest that the pace of removal of the 3' end of the HDRT complementary strand is

321 especially critical, and if the necessary deletion is greater than 10 nucleotides, editing is

322 significantly impaired. These data are consistent with previous observations that Pol $\delta$, the

323 polymerase responsible for 3' extension of the HDRT-associated strand, has modest 3'

324 exonuclease activity. ${ }^{47,48}$ Regardless of the underlying mechanism, this optimization enabled

325 editing efficiencies with Mb2Cas12a and short single-stranded HDRT comparable to those

326 reported with Cas9 and much longer HDRT.

327 With these tools in hand, we showed that the HCDR3 regions of primary human B cells could be 328 reprogrammed to encoded three novel sequences, two of which derived from HIV-1 bNAbs. In 329 each case edited cells could be enriched by FACS with an appropriate antigen while still largely 330 retaining the diversity of the edited repertoire. Interestingly, in the case of cells edited to express 331 the PG9 HCDR, this approach more efficiently enriched BCR encoded by the V3-30 heavy chain 332 than for V3-33, the heavy-chain gene from which the bNAb PG9 originally derived. We 333 confirmed this observation by showing that a PG9 variant constructed from this germline form of 334 this VH gene neutralizes more efficiently than one constructed from the germline V3-33 gene.

335 As importantly, a number of VH genes enriched in this manner bound SOSIP trimers and 
336 neutralized HIV-1. Thus, at least in the case of PG9, a range of modified BCR could respond to a

337 SOSIP antigen or to HIV-1 emerging from a reactivated reservoir.

338 In short, we have overcome several challenges associated with introducing an exogenous

339 HCDR3 sequence into a diverse repertoire of human BCR. In the process we have identified and

340 characterized a Cas12a ortholog especially useful introduced double-strand breaks near the DJ

341 junction of a recombined heavy-chain, and described an optimized approach for replacing

342 genomic regions with short HDRT. Finally, we showed that this approach could create a diverse

343 repertoire of B cells capable of recognizing a critical epitope of HIV-1 Env. These studies

344 establish foundations for proof-of-concept studies in primate models of HIV-1 infection. 


\section{MATERIAL AND METHODS}

347

348

\section{Plasmids}

Wild-type Mb2Cas12a (pcDNA3.1-hMb2Cpf1), Mb3Cas12a (pcDNA3.1-hMb3Cpf1), TsCas12a (pcDNA3.1-hTsCpf1) and BsCas12a (pcDNA3.1-hBsCpf1) plasmids were gifts from Dr. Feng Zhang (Addgene plasmid numbers 69982, 69988, 69983, and 42230, respectively). pMAL-hisLbCpf1-EC was a gift from Dr. Jin-Soo Kim (Addgene plasmid number 79008) and was used to express $\mathrm{Mb} 2 \mathrm{Cas} 12 \mathrm{a}$ in E. coli for protein production. For Cas12a protein production, each Cas12a gene was codon-optimized for E. coli, synthesized by IDT, and cloned into pMAL-hisLbCpf1-EC vector.

\section{Mb2Cas12a protein production and purification}

Expression cassette of maltose binding protein (MBP)-Mb2Cas12a-His in pMal vector was transformed to Rosetta 2 (DE3) (Novagen) competent cells. Single colony was first grown in 5 $\mathrm{mL}$, then scaled up to $10 \mathrm{~L}$ for production, in LB broth with $4 \mu \mathrm{m} / \mathrm{mL}$ chloramphenicol and 100 $\mathrm{ug} / \mathrm{mL}$ carbenicillin. Cell cultures were grown to OD $\sim 0.5$ before placing on ice for $15 \mathrm{~min}$, and added with $0.5 \mathrm{mM}$ IPTG at $16^{\circ} \mathrm{C}$ to induce expression. After $18 \mathrm{hr}$ of incubation, cells were resuspended in the buffer with $50 \mathrm{mM} \mathrm{NaH} 2 \mathrm{PO} 4,500 \mathrm{mM} \mathrm{NaCl}, 15 \mathrm{mM}$ imidazole, 10\% glycerol, and $10 \mathrm{mM}$ Tris at $\mathrm{pH} 8.0$, then sonicated on ice for $20 \mathrm{~min}$ at $18 \mathrm{~W}$ output before clarified by centrifugation for $25 \mathrm{~min}$ at $50,000 \mathrm{~g}$. Clarified supernatant was loaded to the HisTrap FF column (GE Healthcare) and eluted with linear imidazole gradient from $10 \mathrm{mM}$ to $300 \mathrm{mM}$ using ÄKTA explorer (GE Healthcare). To remove the N-terminal MBP tag, the protein elution fractions were pooled and concentrated with $50 \mathrm{kDa}$ molecular weight cutoff ultrafiltration unit (Millipore), then $1 \mathrm{mg}$ of TEV protease was used per $50 \mathrm{mg}$ protein for 
cleavage during dialyzing to the buffer with $250 \mathrm{mM} \mathrm{NaCl}, 0.5 \mathrm{mM}$ EDTA, $1 \mathrm{mM}$ DTT, and 20 mM HEPES with $\mathrm{pH} 7.4$ for $48 \mathrm{~h}$ at $4^{\circ} \mathrm{C}$. For cation exchange chromatography, the protein was diluted with 2-fold volume of 20 mM HEPES with pH 7.0 and loaded on HiTrap SP HP column (GE Healthcare) equilibrated with $100 \mathrm{mM} \mathrm{NaCl}, 20 \mathrm{mM}$ HEPES at $\mathrm{pH}$ 7.0. Proteins were eluted with a linear $\mathrm{NaCl}$ gradient from $100 \mathrm{mM}$ to $2 \mathrm{M}$, then further purified by size exclusion chromatography with Superdex 200 26/60 column (GE Healthcare) with the protein storage buffer (500 mM NaCl, $0.1 \mathrm{mM}$ EDTA, $1 \mathrm{mM}$ DTT, 10\% glycerol and $20 \mathrm{mM}$ HEPES at pH 7.5). Pure protein fractions were pooled and concentrated followed with endotoxin removal with columns from Pierce.

\section{RNP formation and electroporation}

Mb2Cas12a, AsCas12a and SpCas9 gRNAs were ordered from IDT. RNAs were resuspended in Rnase-free water and refolded by incubation at $95^{\circ} \mathrm{C}$ for $5 \mathrm{~min}$ and cooling down at room temperature for $1 \mathrm{~h}$. For each electroporation sample, RNP complexes were formed by mixing $200 \mathrm{pmol}$ of Mb2Cas12a, AsCas12a (Alt-R® A.s. Cas12a (Cpf1) V3 from IDT) or SpCas9 (AltR S.p. Cas9 Nuclease V3 from IDT) with 300 pmol of crRNA and PBS. The RNP mixture was incubated at room temperature for $15 \mathrm{~min}$ to $30 \mathrm{~min}$, then added with $600 \mathrm{pmol}$ of ssDNA HDRT. For the mixture of HDRT (VH1, VH3, and VH4) to target primary cells, 200 pml of each was used. Jeko-1 cells ( 2 million/sample) and human primary B cells (4 million/sample) were harvested and rinsed with PBS before resuspension in electroporation solution. Cells were electroporated using Lonza 4D modules according to Lonza's protocols. After electroporation, cells were incubated in the cuvette for 15 minutes at room temperature before transferring to the antibiotics-free media. Culture media was refreshed in 24 hours. 


\section{HIV protein and antibodies}

BG505 SOSIP v5.2 ds (E64K A316W A73C-A561C I201C-A433C) $)^{41}$ and BG505 E2p ${ }^{43}$ were signal peptide. The apex negative mutant (dBG505) was constructed by altering the V2 basic

PDI (protein disulfide isomerase), respectively (at 4:1:1:1 ratio) using FectoPRO (Polyplus)

402

403

404

405

406

407

408

409

according to the manufacturer's protocol. Supernatants were harvested 5 days after transfection, filtered, and purified with CH01 or PGT145 affinity column. Proteins were eluted with gentle $\mathrm{Ag} / \mathrm{Ab}$ elution buffer (21027, Thermo). The elution was exchanged to buffer (358 mM HEPES, $75 \mathrm{mM} \mathrm{NaCl} \mathrm{pH} \mathrm{8.0)}$. For antibody production, heavy and light chain plasmids were cotransfected (1:1.25 ratio) in Expi293F cells. The supernatants were harvested five days later, and the IgG was purified using protein A Sepharose (GE Healthcare) and eluted with gentle Ag/Ab elution buffer (21027, Thermo), following buffer-exchanged into PBS.

\section{Human cell culture}

Human blood samples were obtained through OneBlood. Peripheral blood mononuclear cells were isolated by density gradient centrifugation with Ficoll (GE Healthcare), stored in liquid nitrogen, then thawed in a $37^{\circ} \mathrm{C}$ water bath and resuspended in human $\mathrm{B}$ cell medium composed of RPMI-1640 with GlutaMAX, supplemented with 10\% FBS or human serum, 10 mM HEPES, 
$1 \mathrm{mM}$ sodium pyruvate, and $53 \mu \mathrm{M} 2$-mercaptoethanol (Gibco). B cells were isolated by magnetic sorting using the Human B Cell Isolation Kit II (130-091-151) from Miltenyi according to the manufacturer's instructions and cultured in the above medium supplemented with $2 \mu \mathrm{g} / \mathrm{ml}$ anti-human RP105 antibody clone (312907, BioLegend).

\section{Flow cytometry and cell sorting}

IgM expression was detected by FITC anti-human IgM antibody (clone MHM-88, Biolegend).

HA insertion was detected by APC anti-HA antibody (clone 16B12, Biolegend). Reprogrammed specificity of B cells were validated by binding with PSG2, SOSIP, E2p proteins conjugated with different fluorophores using Lightning-Link Antibody Labeling Kits according to manufacturer's instructions. Cultured cells were harvested by centrifugation and rinsed by FACS buffer (PBS, 0.5\% BSA, 1 mM EDTA) and resuspend to 10 million/mL in $100 \mu l$. Cells were stained for $20 \mathrm{~min}$ on ice with fluorescently labeled antibodies $(1 \mu \mathrm{g} / \mathrm{mL})$ or proteins $(3$ $\mu \mathrm{g} / \mathrm{mL}$ ), then washed for twice with FACS buffer before measuring fluorescence by BD Accuri C6 flow cytometer or sorting by BD FACSAria Fusion sorter. Flow data were analyzed by FlowJo software.

\section{Sequencing and analysis of the edited B cell IgH repertoire}

Human B cells harvested post gene-editing or cell sorting were lysed for RNA extraction by the RNeasy Micro Kit (74004, Qiagen). Primers used for reverse transcription and library amplification were modified from previous ${ }^{29}$. First-strand cDNA synthesis was performed on 11 $\mu \mathrm{l}$ of total RNA using $10 \mathrm{pmol}$ of each primer in a 20ul total reaction (SuperScript III, Thermo 
437 Fisher) using the manufacturer's protocol. Residual primers and dNTPs were degraded enzymatically (ExoSAP-IT, Thermo Fisher) according to the manufacturer's protocol. Secondstrand synthesis reaction was carried out in $100 \mu \mathrm{l}$ using 10 pmol of each primer (HotStarTaq and dsDNA was purified using 0.8 volumes of SPRI beads (SPRIselect, Beckman Coulter

442 Genomics). 40uL of eluted dsDNA was amplified again with 10 pmol of each primer in a $100 \mu \mathrm{l}$

443 total reaction volume (HotStarTaq Plus). DNA was purified from the PCR reaction product using

$444 \quad 0.8$ volumes of SPRI beads (SPRIselect). $10 \mu \mathrm{l}$ of the eluted PCR product was used in a final

445 indexing using NEBNext Multiplex Oligos for Illumina (E7710S , NEB) following the

447 (SPRIselect). SPRI-purified libraries were sequenced on an Illumina MiSeq using 2×250 bp.

448 Sequencing reads were processed and analyzed. Briefly, paired reads were merged with

449 PANDAseq ${ }^{49}$ using the default merging algorithm then trimmed and collapsed by UMI through

450 Migec using the "checkout" algorithm. Processed reads were mapped by MiGMAP based on

$451 \quad \operatorname{IgBlast}^{50,51}$.

\section{Neutralization assay}

454 Pseudoviruses were produced by co-transfection of different HIV envelope plasmids acquired 455 through the NIH AIDS Reagents Program along with NL4-3- $\Delta$ Env in HEK293T cells using

456 PEIpro (Polyplus). Supernatant was harvested $48 \mathrm{~h}$ post transfection, clarified by centrifugation

457 and $0.45 \mu \mathrm{m}$ filter, and aliquoted for storage at $-80^{\circ} \mathrm{C}$. TZM-bl neutralization assays were

458 performed as previously described ${ }^{52}$. Briefly, titrated antibodies in 96-well plates were incubated 459 with pseudotyped viruses at $37^{\circ} \mathrm{C}$ for 1 hour. TZM-bl cells were then added to the wells with 
50,000 cells/well. Cells were then incubated for 48 hours at $37^{\circ} \mathrm{C}$. At $48 \mathrm{~h}$ post infection, cells were lysed in wells and subjected to Firefly luciferase assays. Viral entry was determined using Britelite Plus (PerkinElmer), and luciferase expression was measured using a Victor X3 plate reader (PerkinElmer).

\section{Statistical analysis}

Data expressed as mean values \pm SD or SEM, and all statistical analysis was performed in GraphPad Prism 7.0 software. $\mathrm{IC}_{50}$ of antibody neutralization was analyzed using default settings for $\log$ (inhibitor) vs. normalized response method. Statistical difference was determined using non-paired Student's t-test or one-way ANOVA with Tukey's test. Differences were considered significant at $\mathrm{P}<0.05$.

\section{ACKNOWLEGEMENTS}

This work is supported by NIH R37 AI091476 and DP1 DA043912 (PI: Farzan). The authors would like to thank Xiaohua $\mathrm{Wu}, \mathrm{Ph} . \mathrm{D}$. for scientific advice on DNA repair mechanisms, and Hyeryun Choe, Ph.D for reviewing of the manuscript. The authors declare they have no financial interests.

\section{AUTHOR CONTRIBUTIONS}

T.O., W.H., G.Z., and M.F. conceived of this study. G.Z. and M.F. provided guidance for experimental design. T.O., W.H., B.Q., Y.G., P.K., H.P., M.D.G., M.H.T., Y.Y., X.Z., and H.W. performed all experiments. T.O., W.H., and M.F. wrote the manuscript. 
FIGURE LEGENDS

483

484

485

486

487

488

489

490

491

492

493

494

495

496

497

498

499

500

501

502

503

504

\section{Figure 1. Targeting the conserved region of JH4 gene requires a Cas12a ortholog}

recognizing non-canonical PAMs. (A) A representation of the coding region of an antibody

heavy-chain variable region is presented. As indicated, the HCDR3 (green) is encoded by the 3'

of a recombined $\mathrm{V}$ gene, a $\mathrm{D}$ gene, and the 5' of a J-chain. To insert a common HCDR3 into a

diverse population of BCR, the guide RNA (gRNA) of a CRISPR effector protein must

complement a conserved $\mathrm{HC}$ region at the 3' of the recombined J-gene, while cleaving a more

variable region near the site of HCDR3 insertion. Note that, unlike Cas9, Cas12a cleaves distally from its PAM and seed regions. The preferred PAM recognition sequence of commonly studied

Cas12a orthologs is TTTV. However, as shown, JH4, the most frequently used JH gene in all species, contains optimally located GTTC and TTCC PAM sequences, located 3' of the HCDR3encoding sequence but oriented Cas12a cleavage within the this sequence. This PAM, sequence of the gRNA, and the Cas12a cut sites are indicated. (B) To identify a Cas12a ortholog efficient at cleaving these non-canonical PAM motifs, the human B-cell line Jeko-1 was transfected with plasmids encoding BsCas12a, TsCas12a, Mb2Cas12a, or Mb3Cas12a. Targeting efficiency was measured by flow cytometry as loss of IgM expression. Among these Cas12a orthologs, Mb2Cas12 most efficiently cleaved the J-chain region initiated with GTTC and TTCC (orange). Error bars indicted range of two independent experiments, and asterisks indicate statistical significance relative to controls. Statistical difference were determined by non-paired Students ttest, $(* * * *, p<0.0001)$. (C) Mb2Cas12 RNP were compared with commercial AsCas12a RNP for their ability cleave four distinct regions in the HCDR3-encoding region of Jeko-1 cells. Loss of IgM expression indicates successful introduction of a double-strand break and inexact NHEJ. (D)

Results of three experiments similar to that shown in panel C. Error bars indicate standard error 
(SEM). Asterisks indicted significant differences from the canonical TTTG PAM (Mb2Cas12aRNP or AsCas12a, respectively). Statistical difference were determined by non-paired Students t-test, $(* * * *, p<0.0001)$.

Figure 2. Optimization of ssDNA templates for Mb2Cas12a-mediated editing the HCDR3encoding region of a human B cell line. (A) A diagram representing four HDR templates (HDRT) used in panels B-E. Specifically, sense and anti-sense forms of HDRT-A, were used to replace a 9-nucleotide (nt) region (grey) with 39-nt insert (green), and both forms of HDRT-B were used to replace a 36-nt region with a 69-nt region. 50-nt homology arms of the sense and antisense forms are represented in red and blue, respectively. SpCas9 (cyan) and Mb2Cas12 (orange) cleavage sites of the target strand (complementary to grNA) are indicated by arrows. Note that paired Cas9 and Cas12a cleavage sites are separated by at most five nucleotides. (B) A representative example of an experiment used to generate panels C-E in which editing efficiency of MbCas12A or SpCas9 RNP is monitored through recognition of an HA tag introduced into the HCDR3 of the Jeko-1 cell BCR by flow cytometry. Control cells were electroporated with Mb2Cas12a RNP without an HDRT. (C) A comparison of Mb2Cas12a (Mb2) and SpCas9 (Cas9) knock-in efficiencies, measured as described in panel B, for all four sites shown in panel A. Differences between Mb2 and Cas9, and among the four sites, are not significant (n.s.). The same data generated for panel $\mathrm{C}$ was replotted according to whether the sense or anti-sense HDRT were used (D), or whether the HDRT complemented the gRNA target or non-target strand. (E) Non-target strand is the PAM containing strand, and the target strand is the strand annealed to gRNA. Again, as indicated, most differences were not significant. However, the HDRT complementary to the Mb2Cas12a gRNA target strand were slightly more efficient than those complementary to the non-target strand $(\mathrm{p}=0.027)$. Dots in $(\mathbf{C})-(\mathbf{E})$ represent pooled data 
from two independent experiments. Statistical significance was calculated by one-way ANOVA with Tukey's multiple comparison test.

\section{Figure 3. The length of the 3 ' mismatch tail determines replacement efficiency with short}

single-stranded HDRT. (A) A model showing where a 3' mismatch tail occurs. A cut site (yellow) is introduced into a region of the gene targeted for replacement (grey), asymmetrically dividing this region. Efficient 5' to 3' resection exposes two 3' ends. An HDRT can complement a strand with a short (left figures) or long 3'-mismatch tail (right figures), which must be removed before the remaining 3' end can be extended to complement the HDRT insert region and its distal homology arm. We propose that the removal of this 3' mismatch tail is a ratelimiting step determining editing efficiency when genomic sequences are replaced. (B) The predicted length of the 3'-mismatch tail in experiments presented in Figure 2 are plotted against the efficiency with which an HA-tag is introduced into the HCDR3 region, as determined by flow cytometry. Error bar indication SD from two independent experiments. (C) A comparison of editing efficiency between those with short $(<10 \mathrm{nt})$ or long $(>10 \mathrm{nt})$ 3' mismatch tails. Editing by SpCas9 or Mb2Cas12a is significantly more efficient with short 3' mismatch tails, as determined by one-way ANOVA with Tukey's multiple comparison test $(p<0.0001)$. Dots represent pool data from two independent experiments.

\section{Figure 4. The BCR specificity of Jeko-1 cells can be reprogrammed with a novel HCDR3.}
(A) The amino-acid sequence of the native Jeko-1 cell HCDR3 region and those of the HIV-1

neutralizing antibodies PG9 and PG16 are shown. In addition the amino-acid translations of four HDRT used in the subsequent panels are represented in green, in the context of the remaining Jeko-1 region. (B) Mb2Cas12a RNP targeting the GTTC PAM of Site 4 in Jeko-1 cells shown in Figure 2B were co-electroporated with the indicated HDRT. Editing efficiency was monitored on 
551 the vertical axis by flow cytometry with fluorescently labeled PSG2, an antibody that recognizes

552 sulfotyrosines within the PG9 and PG16 HCDR3 region, a similarly labeled HIV SOSIP or E2p.

553 The horizontal axis indicates IgM expression, and its loss indicates imprecise NHEJ after

554 Mb2Cas12a-mediated cleavage. Note that introduction of a PG16 HCDR3 was efficient, as

555 indicated by PSG2 recognition, but unlike the PG9 HCDR3, it did not bind the Env trimer. Cells

556 edited to express an HA tag did not bind any reagent. SOSIP proteins were derived from the

557 BG505 HIV-1 isolate. (C) A summary of three independent experiments similar to that shown in

558 panel B. flow cytometric studies used to generate panel B. Error bars indicate SD. (D) Jeko-1

559 edited with PG9-CAR HDRT were enriched by FACS with the anti-sulfotyrosine antibody

560 PSG2. (E) Cells enriched in panel D were analyzed two weeks later by flow cytometry for their

561 ability to bind PSG2, a BG505-based nanoparticle (BG505-E2p), SOSIP trimers derived from

562 the indicated HIV-1 isolate, or an V2 apex negative mutant (dBG505-SOSIP). Grey control

563 indicates wild-type Jeko-1 cells. (F) Unedited Jeko-1 cells and those edited with PG9-CAR

564 HDRT without sorting, or sorted with PSG2 or with E2p, were analyzed by next-generation

565 sequencing (NGS) of the VDJ region. Sequences were divided into four categories, depending on

566 whether the edited sequence matched exactly the HDRT (Perfect HDR), whether HDRT

567 sequence was visible but modified (Imperfect HDR), whether the original Jeko-1 HCDR3 region

568 was intact (Original), or whether this region was modified by NHEJ as indicated by the presence

569 of insertions or deletions (Indel). Representative examples of each category are shown below the

570 charts.

571 Figure 5 Editing primary human B-cells with HDRT recognizing consensus sequences of

572 multiple VH families. (A) A panel of PG9-CAR HDRT with homology arms complementary to

$573 \mathrm{JH} 4$ and to consensus VH1-,VH3-, and VH4-family sequences were evaluated for their ability to 
edited primary human B cells. Cells electroporated with Mb2Cas12a RNP and PG9-CAR HDRT were analyzed by flow cytometry with the anti-sulfotyrosine antibody PSG2 modified with two distinct fluorophores to eliminate non-specific binding from either fluorophore, (B) A summary of results from experiments similar to that shown in panel A, using primary B cells from three independent donors. Note that a mixture of three HDRT edited more cells than any individual HDRT. Null indicates that cells were not electroporated and control indicates cells electroporated with Mb2Cas12a RNP and an HDRT that is not homologous to any sequence in the human genome. Mix indicates cells electroporated with RNP and an equimolar mixture of HDRT with VH1-, VH3- and VH4-specific homology arms. Error bars indicted range of three independent experiments, and asterisks indicate statistical significance calculated by one-way ANOVA with Tukey's multiple comparison test $(*, \mathrm{p}<0.5 ; * *, \mathrm{p}<0.01 ; * * * *, \mathrm{p}<0.0001)$. (C) NGS analysis of primary B cells from two human donors, quantified as described in Figure 4F except that the VH-family of edited cells was also counted.

Figure 6. Reprogrammed primary human B cells retain V-gene diversity. Primary cells were electroporated with Mb2Cas12a RNP and HDRT encoding an HA tag (A) or the HCDR3 regions of the HIV-1 neutralizing antibodies CH01 (B) and PG9 (C), with the same mixture of homology arms as those used in Figure 5. Cells were sorted with an anti-HA antibody (HA tag, panel A) or a SOSIP trimer derived from the CRF_AG_250 isolate (panels B and C). Edited cells were analyzed by NGS before and after sorting, and the frequency of each VH1-,VH3-, and VH4family gene was measured. Flow cytometry histograms displays one of two experiments with similar results, and bar graphs indicate the mean of those two experiments. (D) Antibodies composed the heavy-chains expressed from the indicated $\mathrm{VH}$ genes enriched in panel $\mathrm{C}$ or that of PG9, the PG9 HCDR3, a transmembrane domain, and the native PG9 light chain were 
expressed on the surface of $293 \mathrm{~T}$ cells and analyzed by flow cytometry. One of two representative experiments is presented. Mature indicates expression of the original PG9 antibody. (E) The mean of two experiments shown in panel D is presented. (F) The $\mathrm{IC}_{50}$ values of soluble forms of the antibodies characterized in panel D against indicated HIV-1 isolates is represented.

\section{Reference:}

1. West Jr AP, Scharf L, Scheid JF, Klein F, Bjorkman PJ, Nussenzweig MC. Structural insights on the role of antibodies in HIV-1 vaccine and therapy. Cell. 2014;156(4):633-48.

2. Mascola JR, Haynes BF. HIV-1 neutralizing antibodies: understanding nature's pathways. Immunological reviews. 2013;254(1):225-44.

3. Burton DR, Hangartner L. Broadly neutralizing antibodies to HIV and their role in vaccine design. Annual review of immunology. 2016;34:635-59.

4. Escolano A, Dosenovic P, Nussenzweig MC. Progress toward active or passive HIV-1 vaccination. Journal of Experimental Medicine. 2017;214(1):3-16.

5. Jardine JG, Kulp DW, Havenar-Daughton C, Sarkar A, Briney B, Sok D, Sesterhenn F, EreñoOrbea J, Kalyuzhniy O, Deresa I. HIV-1 broadly neutralizing antibody precursor B cells revealed by germline-targeting immunogen. Science. 2016;351(6280):1458-63.

6. Steichen JM, Lin Y-C, Havenar-Daughton C, Pecetta S, Ozorowski G, Willis JR, Toy L, Sok D, Liguori A, Kratochvil S. A generalized HIV vaccine design strategy for priming of broadly neutralizing antibody responses. Science. 2019;366(6470).

7. Klein F, Diskin R, Scheid JF, Gaebler C, Mouquet H, Georgiev IS, Pancera M, Zhou T, Incesu R-B, Fu BZ. Somatic mutations of the immunoglobulin framework are generally required for broad and potent HIV-1 neutralization. Cell. 2013;153(1):126-38.

8. Walker LM, Huber M, Doores KJ, Falkowska E, Pejchal R, Julien J-P, Wang S-K, Ramos A, Chan-Hui P-Y, Moyle M. Broad neutralization coverage of HIV by multiple highly potent antibodies. Nature. 2011;477(7365):466-70.

9. Huang D, Tran JT, Olson A, Vollbrecht T, Tenuta M, Guryleva MV, Fuller RP, Schiffner T, Abadejos JR, Couvrette L. Vaccine elicitation of HIV broadly neutralizing antibodies from engineered B cells. Nature communications. 2020;11(1):1-10.

10. Voss JE, Gonzalez-Martin A, Andrabi R, Fuller RP, Murrell B, McCoy LE, Porter K, Huang D, Li W, Sok D. Reprogramming the antigen specificity of B cells using genome-editing technologies. Elife. 2019;8:e42995.

11. Hartweger H, McGuire AT, Horning M, Taylor JJ, Dosenovic P, Yost D, Gazumyan A, Seaman MS, Stamatatos L, Jankovic M. HIV-specific humoral immune responses by CRISPR/Cas9-edited B cells. Journal of Experimental Medicine. 2019;216(6):1301-10. 
12. Moffett HF, Harms CK, Fitzpatrick KS, Tooley MR, Boonyaratanakornkit J, Taylor JJ. B cells engineered to express pathogen-specific antibodies protect against infection. Science immunology. 2019;4(35).

13. Nahmad AD, Raviv Y, Horovitz-Fried M, Sofer I, Akriv T, Nataf D, Dotan I, Carmi Y, Burstein D, Wine Y. Engineered B cells expressing an anti-HIV antibody enable memory retention, isotype switching and clonal expansion. Nature communications. 2020;11(1):1-10. 14. Giudicelli V, Duroux P, Ginestoux C, Folch G, Jabado-Michaloud J, Chaume D, Lefranc MP. IMGT/LIGM-DB, the IMGT ${ }^{\circledR}$ comprehensive database of immunoglobulin and $\mathrm{T}$ cell receptor nucleotide sequences. Nucleic acids research. 2006;34(supp1_1):D781-D4.

15. Rolink A, Melchers F. Molecular and cellular origins of B lymphocyte diversity. Cell. 1991;66(6):1081-94.

16. Elhanati Y, Sethna Z, Marcou Q, Callan Jr CG, Mora T, Walczak AM. Inferring processes underlying B-cell repertoire diversity. Philosophical Transactions of the Royal Society B: Biological Sciences. 2015;370(1676):20140243.

17. Schatz DG, Ji Y. Recombination centres and the orchestration of V (D) J recombination. Nature Reviews Immunology. 2011;11(4):251-63.

18. Bassing $\mathrm{CH}$, Swat W, Alt FW. The mechanism and regulation of chromosomal V (D) J recombination. Cell. 2002;109(2):S45-S55.

19. Mesin L, Ersching J, Victora GD. Germinal center B cell dynamics. Immunity. 2016;45(3):471-82.

20. Leslie A, Pfafferott K, Chetty P, Draenert R, Addo M, Feeney M, Tang Y, Holmes E, Allen T, Prado J. HIV evolution: CTL escape mutation and reversion after transmission. Nature medicine. 2004;10(3):282-9.

21. Abbott RK, Lee JH, Menis S, Skog P, Rossi M, Ota T, Kulp DW, Bhullar D, Kalyuzhniy O, Havenar-Daughton C. Precursor frequency and affinity determine B cell competitive fitness in germinal centers, tested with germline-targeting HIV vaccine immunogens. Immunity. 2018;48(1):133-46. e6.

22. Dosenovic P, Kara EE, Pettersson A-K, McGuire AT, Gray M, Hartweger H, Thientosapol ES, Stamatatos L, Nussenzweig MC. Anti-HIV-1 B cell responses are dependent on B cell precursor frequency and antigen-binding affinity. Proceedings of the National Academy of Sciences. 2018;115(18):4743-8.

23. Andrabi R, Voss JE, Liang C-H, Briney B, McCoy LE, Wu C-Y, Wong C-H, Poignard P, Burton DR. Identification of common features in prototype broadly neutralizing antibodies to HIV envelope V2 apex to facilitate vaccine design. Immunity. 2015;43(5):959-73.

24. Lee JH, Andrabi R, Su C-Y, Yasmeen A, Julien J-P, Kong L, Wu NC, McBride R, Sok D, Pauthner M. A broadly neutralizing antibody targets the dynamic HIV envelope trimer apex via a long, rigidified, and anionic $\beta$-hairpin structure. Immunity. 2017;46(4):690-702.

25. Zetsche B, Strecker J, Abudayyeh OO, Gootenberg JS, Scott DA, Zhang F. A Survey of Genome Editing Activity for 16 Cpf1 orthologs. bioRxiv. 2017:134015.

26. Doudna JA, Charpentier E. The new frontier of genome engineering with CRISPR-Cas9. Science. 2014;346(6213).

27. Wang Y, Liu KI, Sutrisnoh N-AB, Srinivasan H, Zhang J, Li J, Zhang F, Lalith CRJ, Xing H, Shanmugam R. Systematic evaluation of CRISPR-Cas systems reveals design principles for genome editing in human cells. Genome biology. 2018;19(1):1-16. 
28. Liang X, Potter J, Kumar S, Ravinder N, Chesnut JD. Enhanced CRISPR/Cas9-mediated precise genome editing by improved design and delivery of gRNA, Cas9 nuclease, and donor DNA. Journal of biotechnology. 2017;241:136-46.

29. Briney B, Inderbitzin A, Joyce C, Burton DR. Commonality despite exceptional diversity in the baseline human antibody repertoire. Nature. 2019;566(7744):393-7.

30. Volpe JM, Kepler TB. Large-scale analysis of human heavy chain V (D) J recombination patterns. Immunome research. 2008;4(1):1-10.

31. Zetsche B, Gootenberg JS, Abudayyeh OO, Slaymaker IM, Makarova KS, Essletzbichler P, Volz SE, Joung J, Van Der Oost J, Regev A. Cpf1 is a single RNA-guided endonuclease of a class 2 CRISPR-Cas system. Cell. 2015;163(3):759-71.

32. Jinek M, Chylinski K, Fonfara I, Hauer M, Doudna JA, Charpentier E. A programmable dualRNA-guided DNA endonuclease in adaptive bacterial immunity. science. 2012;337(6096):81621.

33. Yamano T, Zetsche B, Ishitani R, Zhang F, Nishimasu H, Nureki O. Structural basis for the canonical and non-canonical PAM recognition by CRISPR-Cpf1. Molecular cell. 2017;67(4):63345. e3.

34. Shalem O, Sanjana NE, Hartenian E, Shi X, Scott DA, Mikkelsen TS, Heckl D, Ebert BL, Root DE, Doench JG. Genome-scale CRISPR-Cas9 knockout screening in human cells. Science. 2014;343(6166):84-7.

35. Hur JK, Kim K, Been KW, Baek G, Ye S, Hur JW, Ryu S-M, Lee YS, Kim J-S. Targeted mutagenesis in mice by electroporation of $\mathrm{Cpf1}$ ribonucleoproteins. Nature biotechnology. 2016;34(8):807-8.

36. Paix A, Folkmann A, Goldman DH, Kulaga H, Grzelak MJ, Rasoloson D, Paidemarry S, Green R, Reed RR, Seydoux G. Precision genome editing using synthesis-dependent repair of Cas9induced DNA breaks. Proceedings of the National Academy of Sciences. 2017;114(50):E10745E54.

37. Richardson CD, Ray GJ, DeWitt MA, Curie GL, Corn JE. Enhancing homology-directed genome editing by catalytically active and inactive CRISPR-Cas9 using asymmetric donor DNA. Nature biotechnology. 2016;34(3):339-44.

38. Walker LM, Phogat SK, Chan-Hui P-Y, Wagner D, Phung P, Goss JL, Wrin T, Simek MD, Fling S, Mitcham JL. Broad and potent neutralizing antibodies from an African donor reveal a new HIV-1 vaccine target. Science. 2009;326(5950):285-9.

39. Sok D, van Gils MJ, Pauthner M, Julien J-P, Saye-Francisco KL, Hsueh J, Briney B, Lee JH, Le KM, Lee PS. Recombinant HIV envelope trimer selects for quaternary-dependent antibodies targeting the trimer apex. Proceedings of the National Academy of Sciences. 2014;111(49):176249.

40. Sanders RW, Derking R, Cupo A, Julien J-P, Yasmeen A, de Val N, Kim HJ, Blattner C, de la Peña AT, Korzun J. A next-generation cleaved, soluble HIV-1 Env trimer, BG505 SOSIP. 664 gp140, expresses multiple epitopes for broadly neutralizing but not non-neutralizing antibodies. PLoS pathog. 2013;9(9):e1003618.

41. de la Peña AT, Julien J-P, de Taeye SW, Garces F, Guttman M, Ozorowski G, Pritchard LK, Behrens A-J, Go EP, Burger JA. Improving the immunogenicity of native-like HIV-1 envelope trimers by hyperstabilization. Cell reports. 2017;20(8):1805-17.

42. Hoffhines AJ, Damoc E, Bridges KG, Leary JA, Moore KL. Detection and purification of tyrosine-sulfated proteins using a novel anti-sulfotyrosine monoclonal antibody. Journal of Biological Chemistry. 2006;281(49):37877-87. 
43. He L, De Val N, Morris CD, Vora N, Thinnes TC, Kong L, Azadnia P, Sok D, Zhou B, Burton DR. Presenting native-like trimeric HIV-1 antigens with self-assembling nanoparticles. Nature communications. 2016;7(1):1-15.

726 44. Briney B, Sok D, Jardine JG, Kulp DW, Skog P, Menis S, Jacak R, Kalyuzhniy O, De Val N, 727 Sesterhenn F. Tailored immunogens direct affinity maturation toward HIV neutralizing antibodies. 728 Cell. 2016;166(6):1459-70. e11.

729 45. Escolano A, Steichen JM, Dosenovic P, Kulp DW, Golijanin J, Sok D, Freund NT, Gitlin AD, 730 Oliveira T, Araki T. Sequential immunization elicits broadly neutralizing anti-HIV-1 antibodies in 731 Ig knockin mice. Cell. 2016;166(6):1445-58. e12.

732 46. Tian M, Cheng C, Chen X, Duan H, Cheng H-L, Dao M, Sheng Z, Kimble M, Wang L, Lin S. 733 Induction of HIV neutralizing antibody lineages in mice with diverse precursor repertoires. Cell. 734 2016;166(6):1471-84. e18.

735 47. Pâques F, Haber JE. Two pathways for removal of nonhomologous DNA ends during double736 strand break repair in Saccharomyces cerevisiae. Molecular and Cellular Biology. 1997;17(11):6765-71.

738 48. Anand R, Beach A, Li K, Haber J. Rad51-mediated double-strand break repair and mismatch correction of divergent substrates. Nature. 2017;544(7650):377-80. 49. Masella AP, Bartram AK, Truszkowski JM, Brown DG, Neufeld JD. PANDAseq: paired-end assembler for illumina sequences. BMC bioinformatics. 2012;13(1):1-7. 50. Shugay M, Britanova OV, Merzlyak EM, Turchaninova MA, Mamedov IZ, Tuganbaev TR, Bolotin DA, Staroverov DB, Putintseva EV, Plevova K. Towards error-free profiling of immune repertoires. Nature methods. 2014;11(6):653-5.

745 51. Shugay M, Bagaev DV, Turchaninova MA, Bolotin DA, Britanova OV, Putintseva EV, 746 Pogorelyy MV, Nazarov VI, Zvyagin IV, Kirgizova VI. VDJtools: unifying post-analysis of T cell 747 receptor repertoires. PLoS computational biology. 2015;11(11):e1004503.

748 52. Montefiori DC. Measuring HIV neutralization in a luciferase reporter gene assay. HIV 749 protocols: Springer; 2009. p. 395-405. 
bioRxiv preprint doi: https://doi.org/10.1101/2021.04.01.437943; this version posted April 3, 2021. The copyright holder for this preprint (which was not certified by peer review) is the author/funder, who has granted bioRxiv a license to display the preprint in perpetuity. It is made available under aCC-BY-NC-ND 4.0 International license.

\section{Figure 1}

A

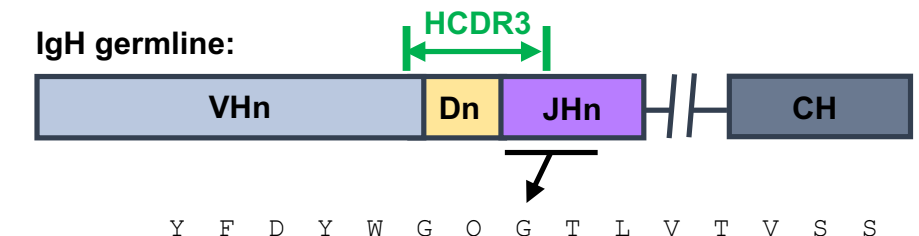

JH4 ... GATGAAACTGATGACCCCGGTTCCTTGGACCAGTGGCAGAGGAGTC...

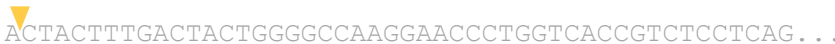

JH4

VACTACTITGACTACTGGGGCCAAGGACCCTGGTCACCGTCTCCTCAG... TGATGA

Cut site

gRNA PAM

C

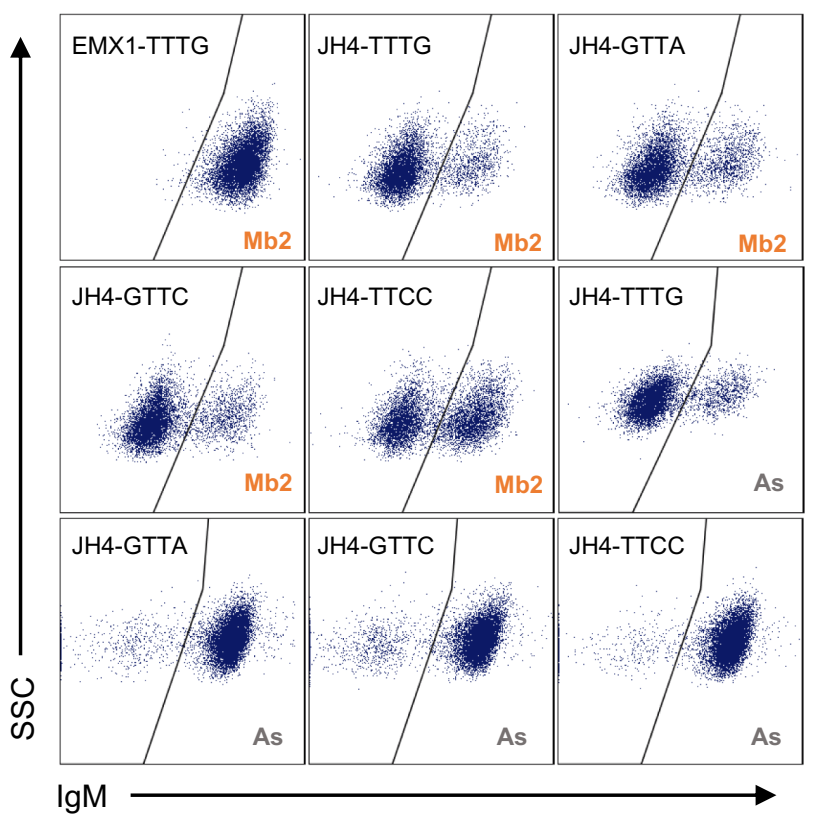

B

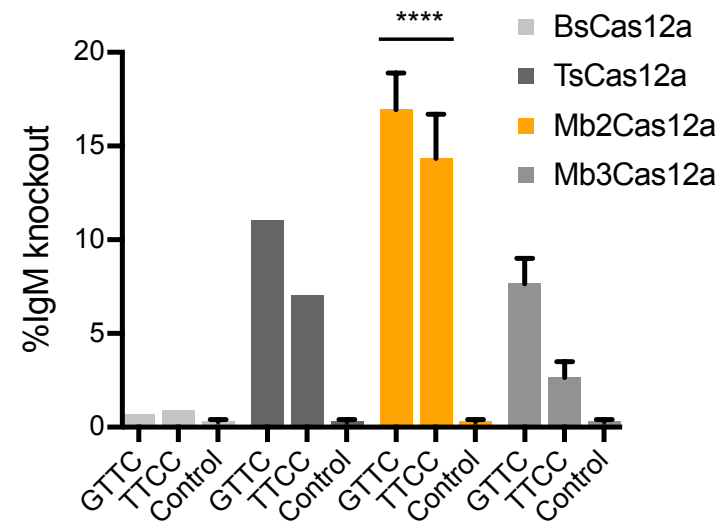

D

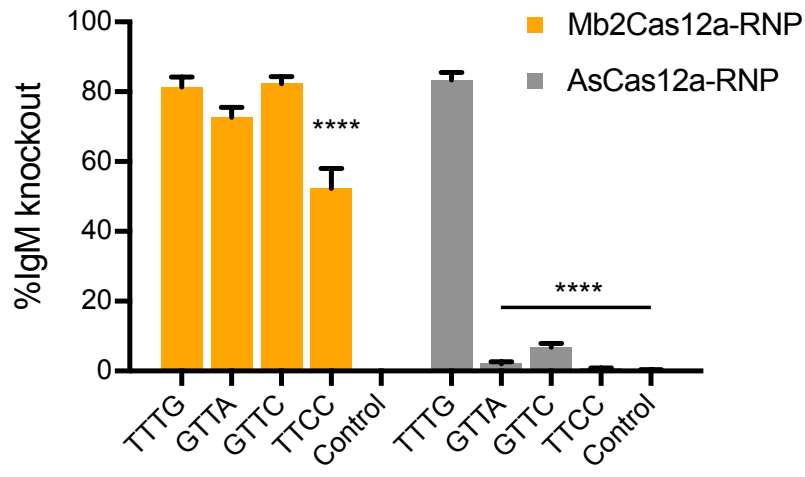


bioRxiv preprint doi: https://doi.org/10.1101/2021.04.01.437943; this version posted April 3, 2021. The copyright holder for this preprint (which was not certified by peer review) is the author/funder, who has granted bioRxiv a license to display the preprint in perpetuity. It is made available under ACC-BY-NC-ND 4.0 International license.

\section{Figure 2}

A

HDRT-A:

sense 5 ' - 50nt 39 nt 50nt

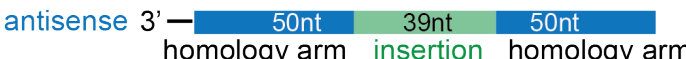

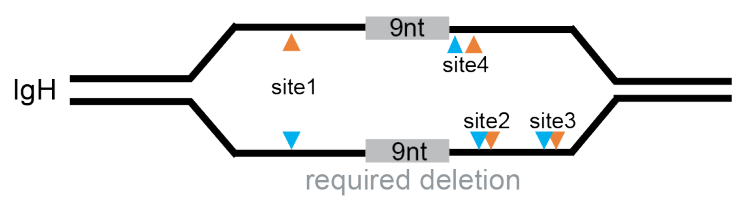

HDRT-B:

\begin{tabular}{ccc} 
sense $5^{\prime}-50 n t$ & $69 n t$ & $50 n t$ \\
\hline $50 n t$ & $69 n t$ & $50 n t$ \\
\hline
\end{tabular} homology arm insertion homology arm

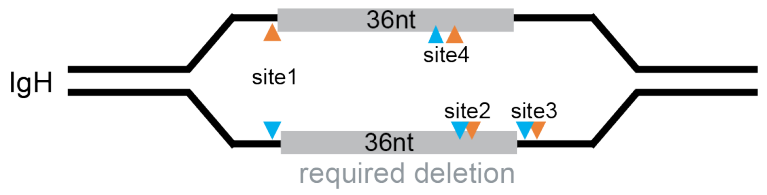

$\Delta$ SpCas9-cut site of target strand

$\triangle \mathrm{Mb2Cas} 12 \mathrm{a}$-cut site of target strand
B

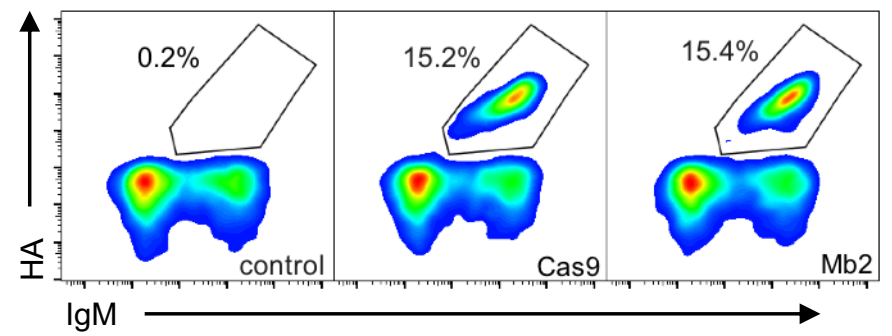

C

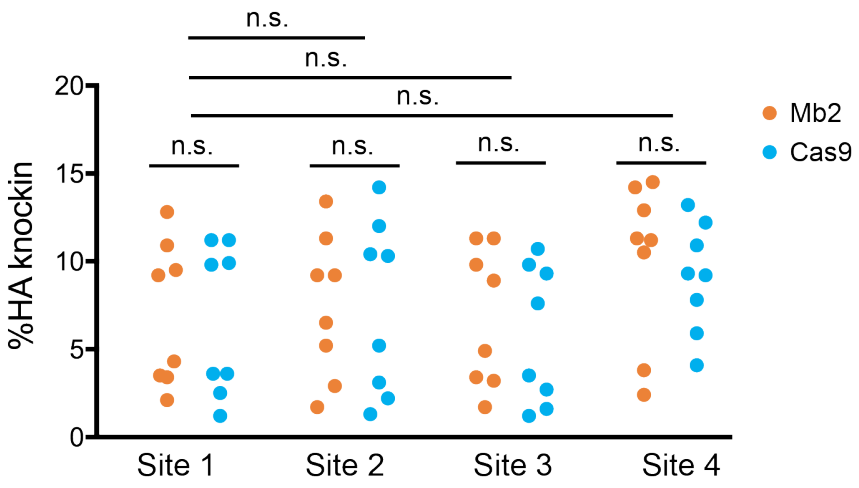

D

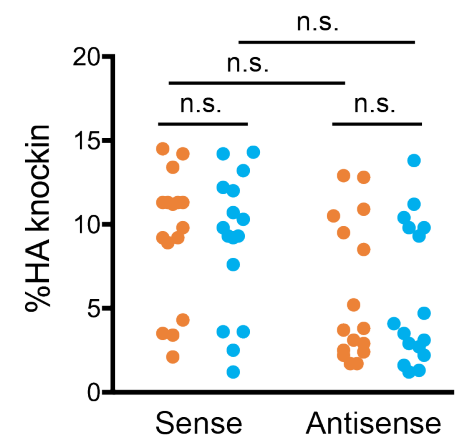

E

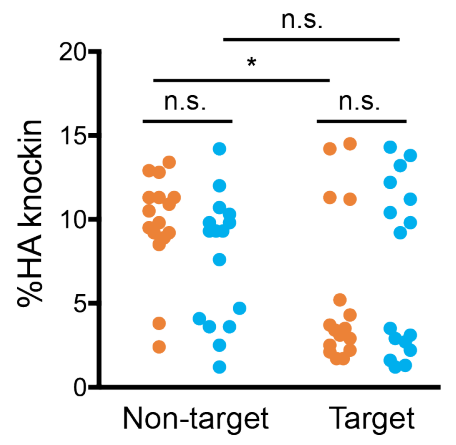


bioRxiv preprint doi: https://doi.org/10.1101/2021.04.01.437943; this version posted April 3, 2021. The copyright holder for this preprint (which was not certified by peer review) is the author/funder, who has granted bioRxiv a license to display the preprint in perpetuity. It is made available under aCC-BY-NC-ND 4.0 International license.

\section{Figure 3}

A

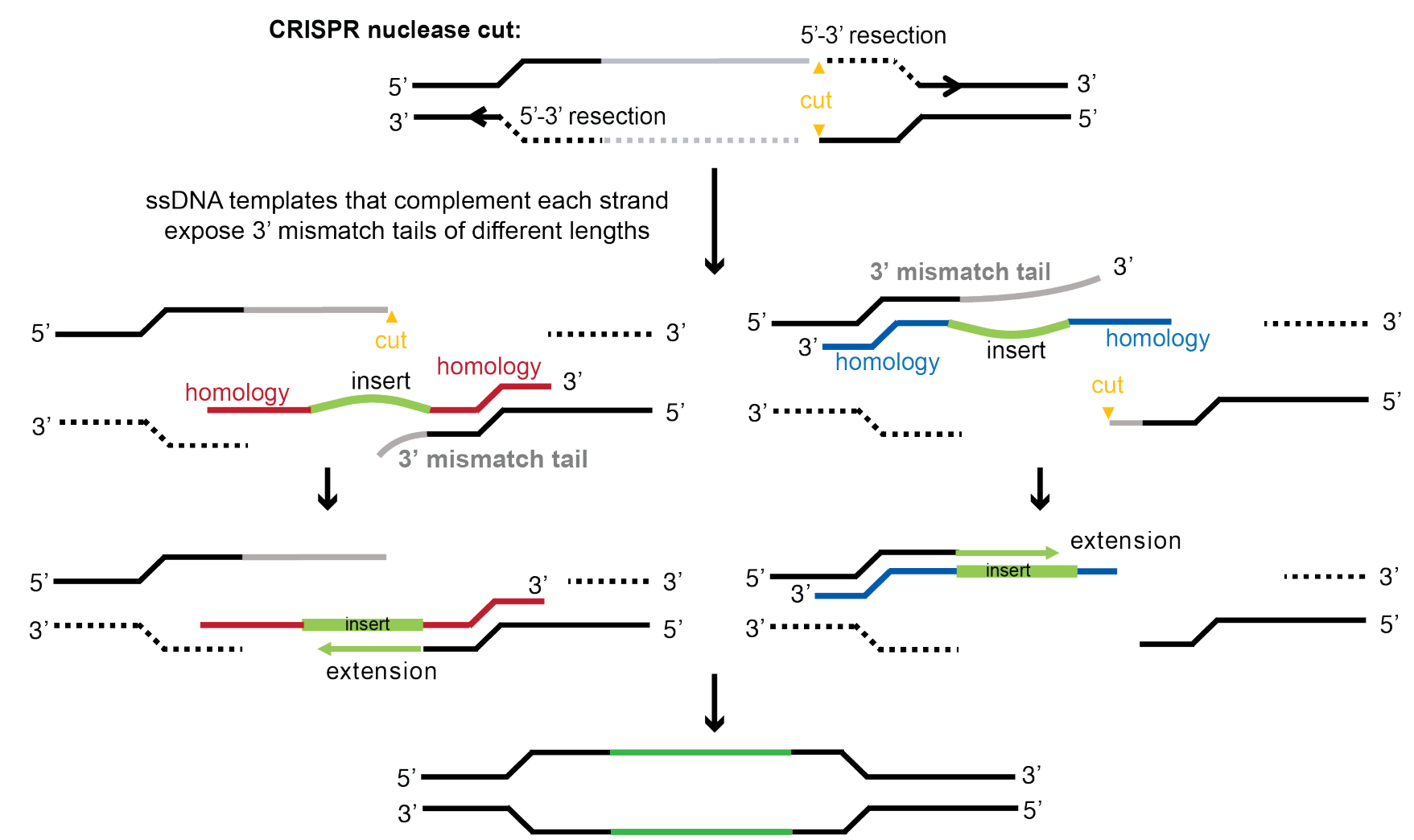

B

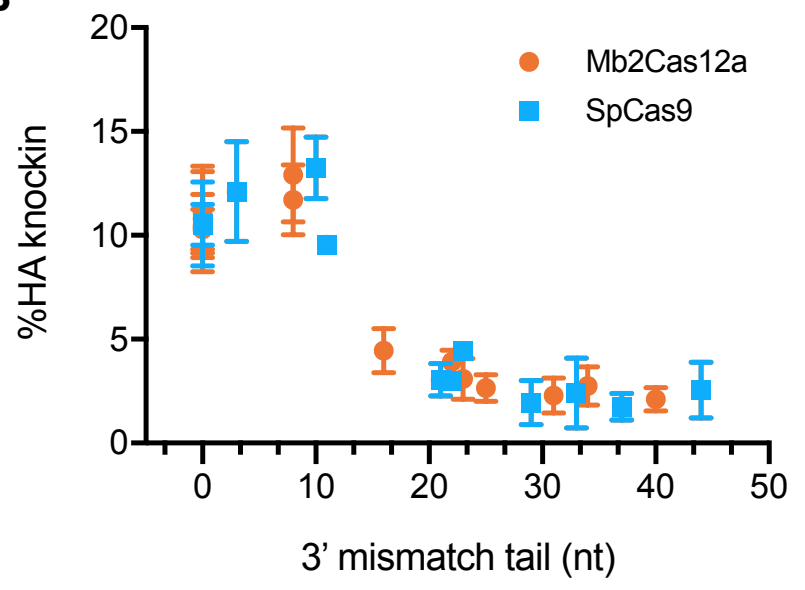

C

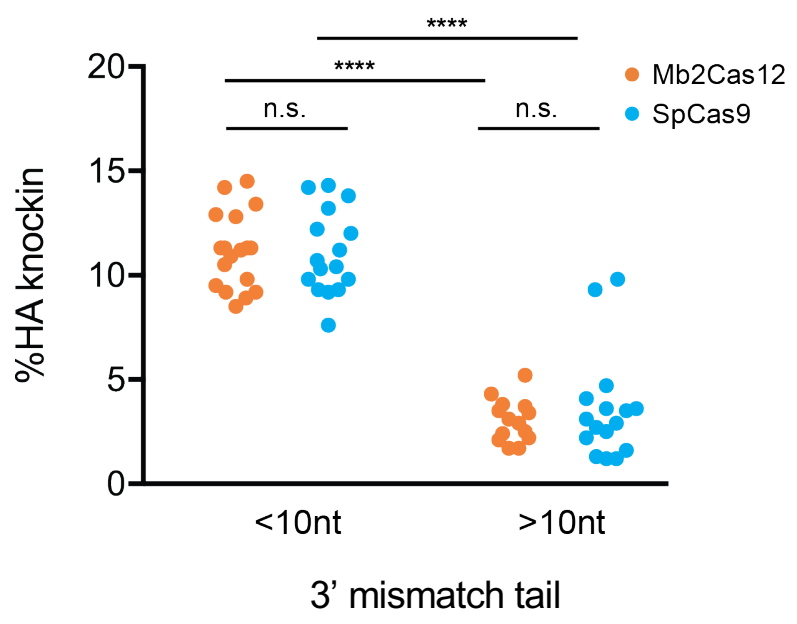


bioRxiv preprint doi: https://doi.org/10.1101/2021.04.01.437943; this version posted April 3, 2021. The copyright holder for this preprint (which was not certified by peer review) is the author/funder, who has granted bioRxiv a license to display the preprint in perpetuity. It is made available under aCC-BY-NC-ND 4.0 International license.

\section{Figure 4}

A HCDR3 sequence:

Jeko-1 CARIRGFGVVNLPDYWGQG

PG9 CVREAGGPDYRNGYNYYDFYDGYYNYHYMDVWGKG

PG16 CAREAGGPIWHDDVKYYDFNDGYYNYHYMDVWGQG

HDRT:

PG9-CVR CVREAGGPDYRNGYNYYDFYDGYYNYHYMDYWGQG

PG9-CAR CAREAGGPDYRNGYNYYDFYDGYYNYHYMDYWGOG

PG16 CAREAGGPIWHDDVKYYDFNDGYYNYHYMDYWGQG

HA tag CARGGAGGYPYDVPDYAGGAGGYFDYWGQG

C

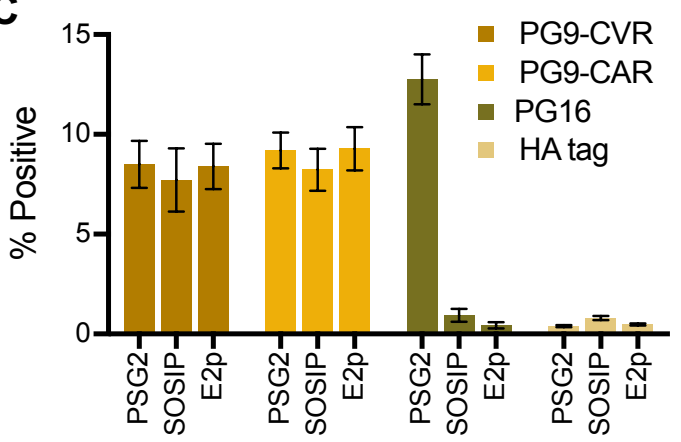

B

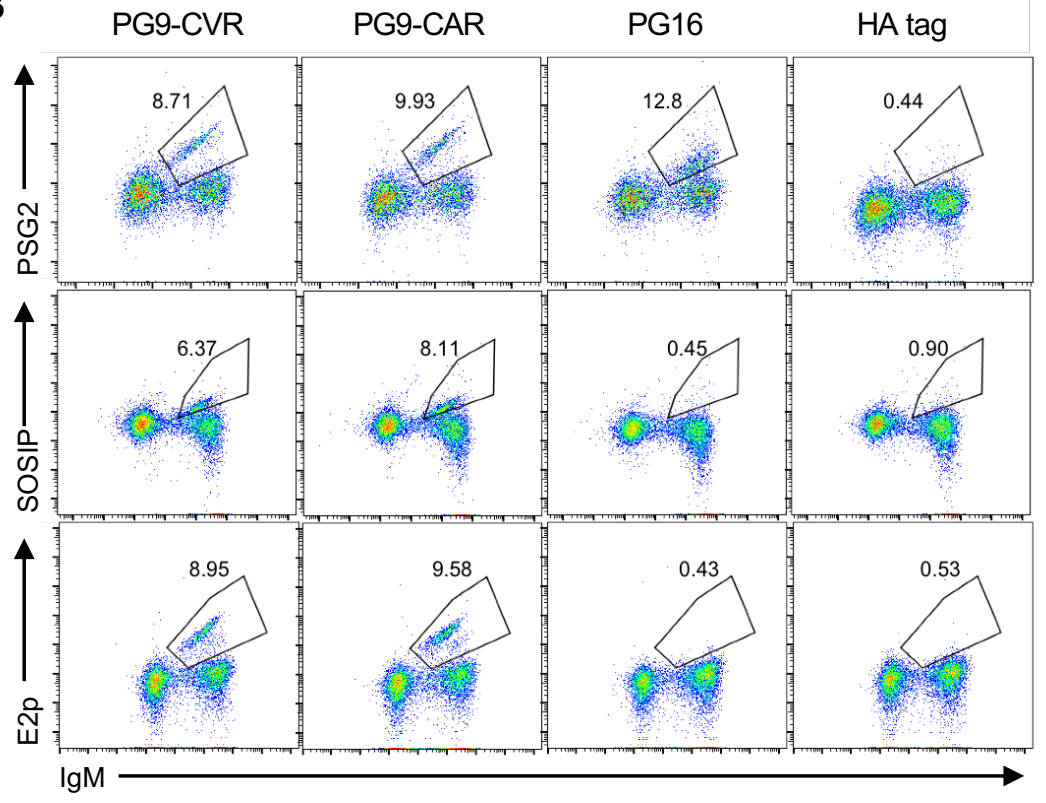

$\mathbf{F}$

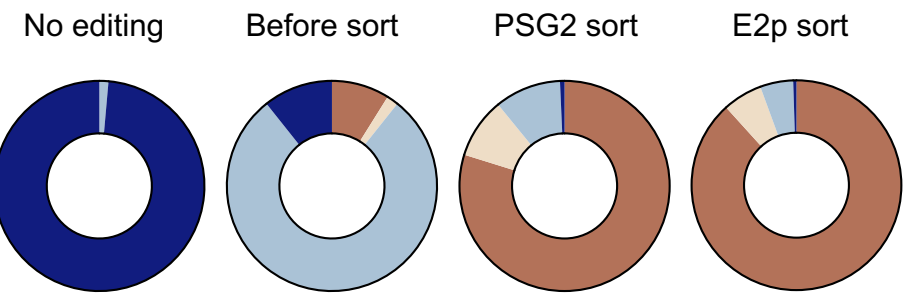

E
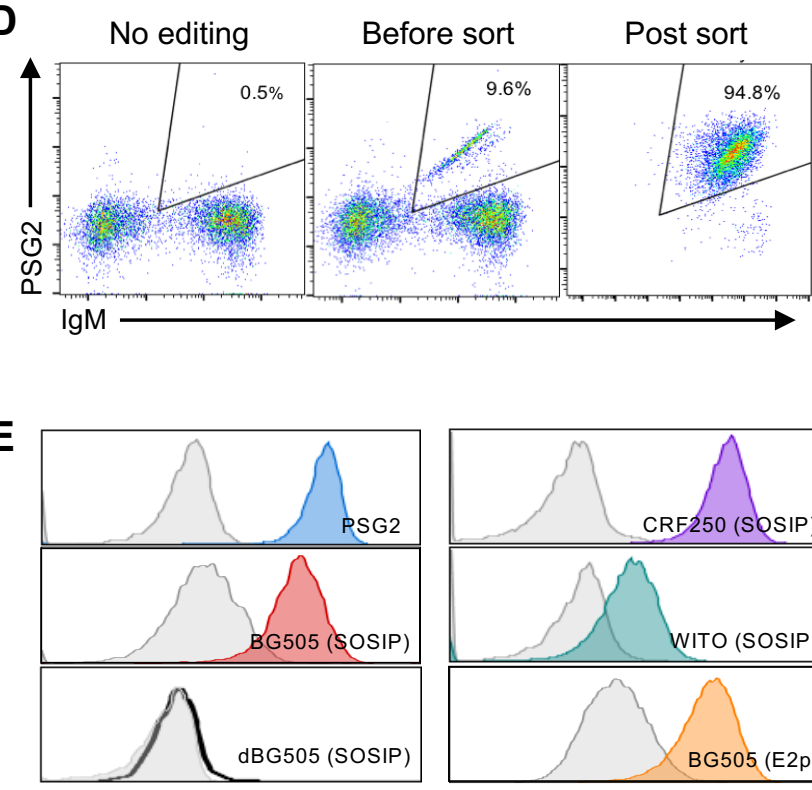

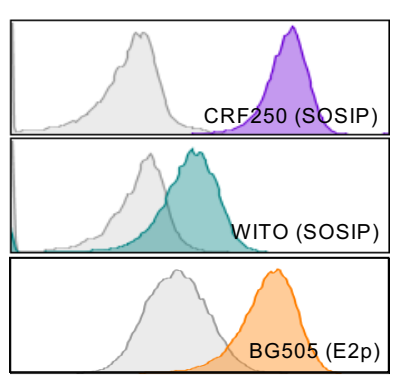

Representative sequences:

\begin{tabular}{|l|l|}
\hline CARIRGFGVVNLPDYW & Original \\
\hline CARIGVVNLPDHW & Indel \\
\hline CAREAGGPDYRNGYNYYDFYDGYYNYHYMDYW & Perfect HDR \\
\hline CAREAGGPDYRNGYNYYLPDYW & Imperfect HDR \\
\hline CAREAGGPDYRNGYSYYDFYDGYYNYHYMDYW & Imperfect HDR \\
\hline CAREAGGPDYRNGYNYYDFYDGYYNWGFGVVNLPDYW & Imperfect HDR \\
\hline
\end{tabular}


bioRxiv preprint doi: https://doi.org/10.1101/2021.04.01.437943; this version posted April 3, 2021. The copyright holder for this preprint (which was not certified by peer review) is the author/funder, who has granted bioRxiv a license to display the preprint in perpetuity. It is made available under aCC-BY-NC-ND 4.0 International license.

Figure 5

A

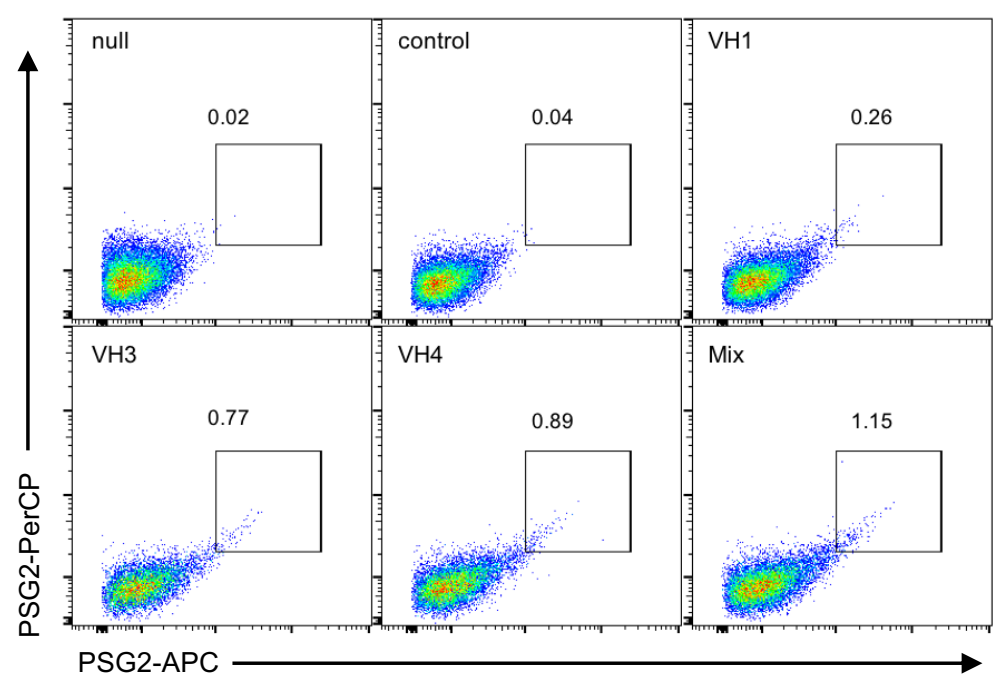

B

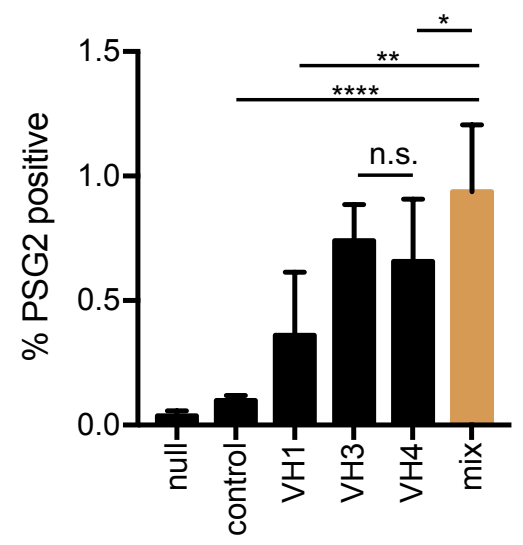

C

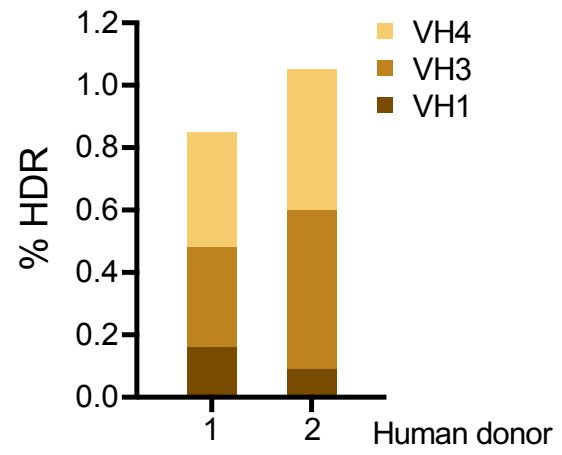


bioRxiv preprint doi: https://doi.org/10.1101/2021.04.01.437943; this version posted April 3, 2021. The copyright holder for this preprint (which

was not certified by peer review) is the author/funder, who has granted bioRxiv a license to display the preprint in perpetuity. It is made available under aCC-BY-NC-ND 4.0 International license.

Figure 6

A

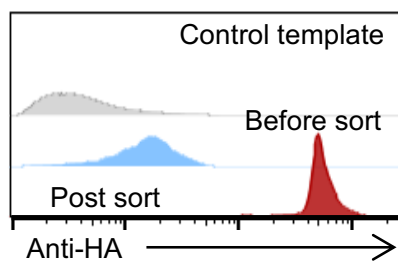

B

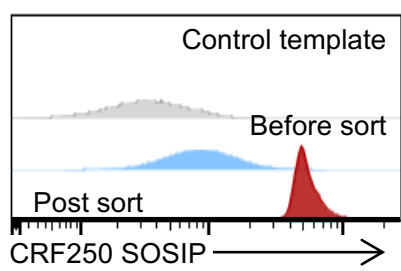

C

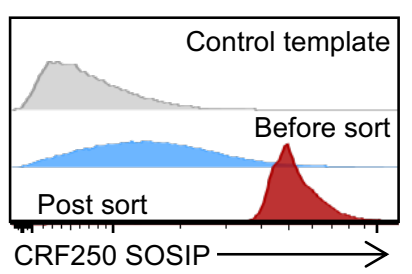

HA-HCDR3
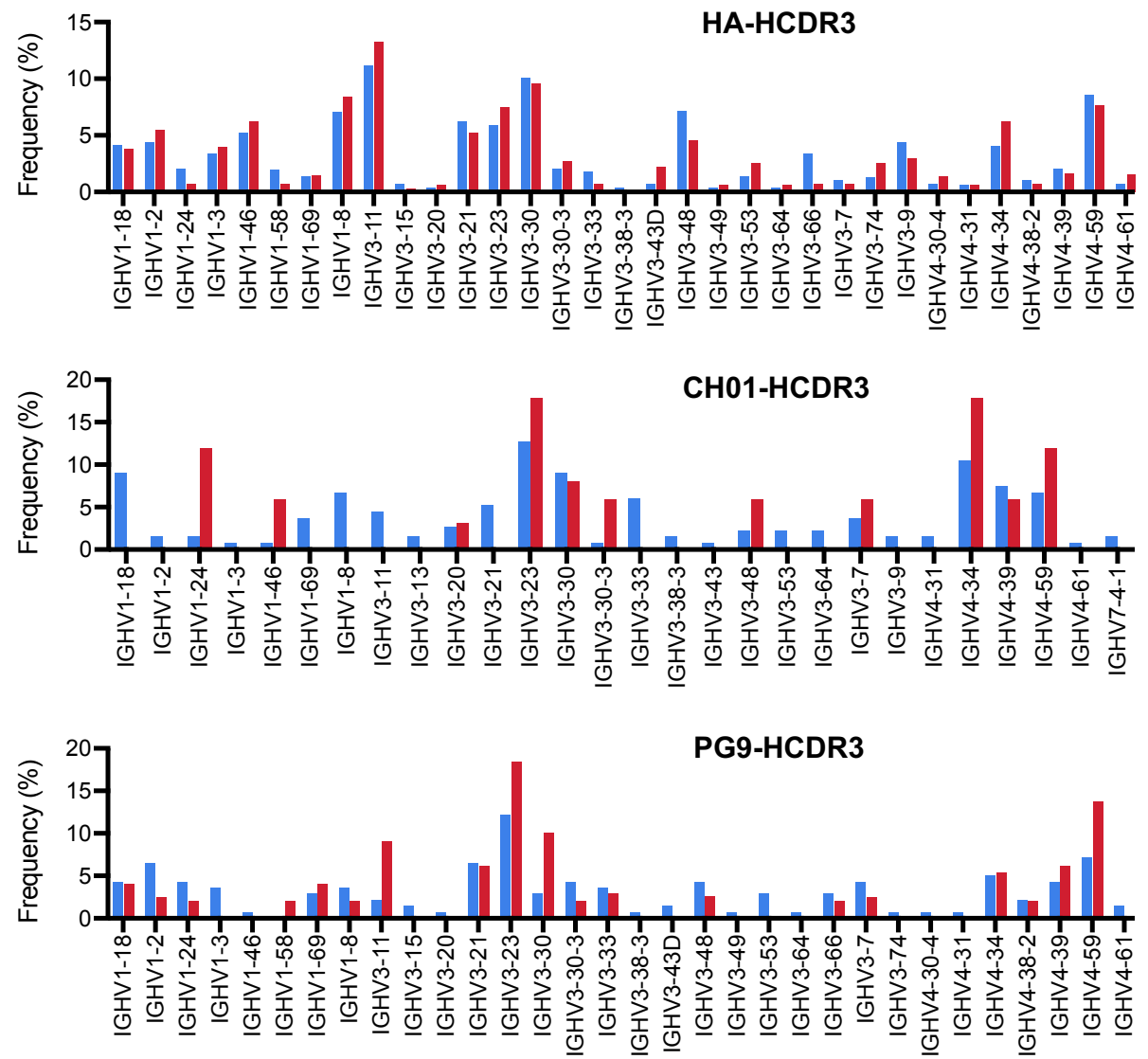

D

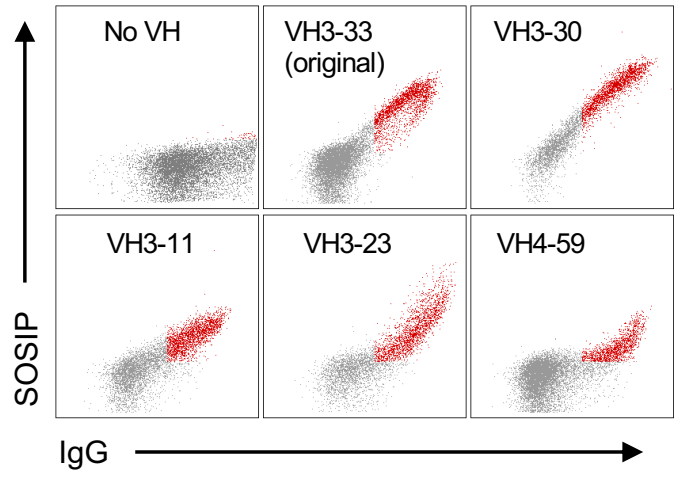

E

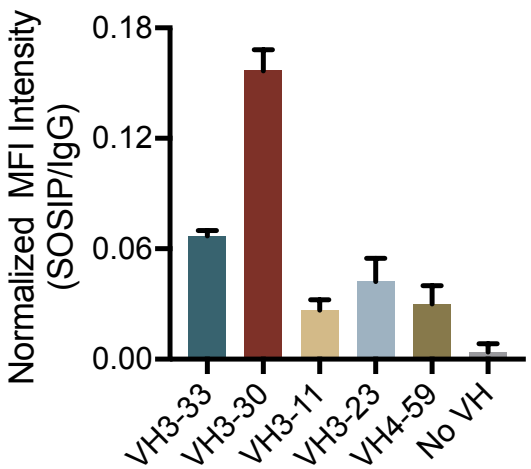

F

\begin{tabular}{|c|c|c|c|c|c|c|}
\hline HIV isolate & Clade & VH3-33 & VH3-30 & VH3-23 & VH3-11 & VH4-59 \\
\hline $\mathbf{1 6 0 5 5}$ & C & 1.81 & 0.95 & 1.67 & 3.99 & 3.15 \\
\hline $\mathbf{2 5 7 1 0}$ & C & 34.94 & 4.89 & $>50$ & $>50$ & $>50$ \\
\hline Bal.26 & B & $>50$ & 2.30 & $>50$ & $>50$ & $>50$ \\
\hline BG505 & A & 8.02 & 2.29 & 32.22 & $>50$ & $>50$ \\
\hline CRF_AG_250 & AG & 0.57 & 0.20 & 0.86 & 4.20 & 1.49 \\
\hline
\end{tabular}

